\title{
A New Peak-to-Average Power-Ratio Reduction Algorithm for OFDM Systems via Constellation Extension
}

\author{
Y. J. Kou, W.-S. Lu, Fellow, IEEE and A. Antoniou Fellow, IEEE
}

\begin{abstract}
Peak-to-average power-ratio (PAPR) reduction for OFDM systems is investigated in a probabilistic framework. A new constellation extension technique is developed whereby the data for each subcarrier are represented either by points in the original constellation or by extended points. An optimal representation of the OFDM signal is achieved by using a de-randomization algorithm where the conditional probability involved is handled by using the Chernoff bound and the evaluation of the many hyperbolic cosine functions involved is replaced by a tight upper bound for these functions. The proposed algorithm can be used by itself or be combined with a selective rotation technique described in the paper and with other known algorithms such as the coordinate descent optimization and selective mapping algorithms to achieve further performance enhancements at the cost of a slight increase in the computational complexity. When compared with other existing PAPR-reduction algorithms, the enhanced algorithm offers improved PAPRreduction performance and improved computational complexity although the transmit power is increased somewhat.
\end{abstract}

Index Terms-OFDM, peak-to-average power-ratio reduction, constellation extension, de-randomization.

\section{INTRODUCTION}

The demand for high data-rate services over wireless networks has been increasing very rapidly in recent years. These services require reliable data transmission over band-limited wireless channels which are subjected to many degradations such as noise, multipath fading, and interference. A transmission technique that offers robustness with respect to multipath fading and resistance to narrowband interference is orthogonal frequency-division multiplexing (OFDM) [1]-[3]. Because of its many advantages, OFDM has gained much popularity and has been used widely in a variety of communication systems. Well-known examples include digital audio broadcasting [4], digital video broadcasting [5], and the IEEE 802.11a and 802.11g standards for wireless local area networks [6].

A major drawback associated with OFDM is its large peakto-average power-ratio (PAPR) which makes system performance very sensitive to distortion introduced by nonlinear devices such as power amplifiers (PAs) [1]-[3][7][8]. In an attempt to reduce the nonlinear distortion caused by the PAs, several techniques have been proposed that can reduce the PAPR of the OFDM signal before it enters a PA. A straightforward technique would be to limit the signal strength at the transmitter to a desired level through clipping but this technique degrades the bit-error-rate (BER) of the system and increases the out-of-band radiation [9][10] due to the increased harmonic content. Coding and bandpass filtering can reduce these effects but, unfortunately, they would increase the cost of the system [11]. A more efficient approach for the reduction of nonlinear distortion is through the use of PAPR-reduction algorithms and a variety of such algorithms have been described in the literature [12]-[28]. In [12]-[17], algorithms are described which combine PAPR reduction with error-control coding. Very low PAPR can be achieved by these algorithms but at the cost of a significant reduction in the data transmission rate. Moreover, these algorithms require large look-up tables and, therefore, are more suitable for OFDM systems with a small number of subcarriers. A multiple signal representation approach has been proposed in [18][19] where a set of OFDM signals are generated at the OFDM transmitter and the transmit signal with the lowest peak power is selected. This approach is computationally efficient but it requires the transmission of a small amount of side information. The use of the selective mapping (SM) algorithm [18] together with other PAPR-reduction algorithms has been proposed in [20]. In [21]-[22], PAPR reduction has been achieved by using trellis shaping at the cost of an increase in the decoding complexity of the receiver. In [23]-[28], reduced PAPR is achieved by extending the modulation constellation. These algorithms offer distortionless PAPR reduction and do not require the transmission of any side information. The algorithms differ from one another in the way they modify the modulation constellation. In [23], a tone reservation algorithm has been developed where several subcarriers are set aside for PAPR reduction. Since the subcarriers are orthogonal, the additive signal on unused subcarriers causes no distortion to the databearing subcarriers. In [24], a tone injection algorithm has been developed where the constellation points in a number of subcarriers are modified to achieve PAPR reduction at the cost of a slight increase in transmit power. In [25][26], an active set extension (ASE) algorithm has been proposed where PAPR reduction is achieved by modifying the exterior modulation constellation over active subcarriers in a way that will not degrade the BER performance. In [27], a symmetric constellation extension (SCE) algorithm has been proposed for PAPR reduction whereby the subsymbols for each subcarrier are represented by two symmetric constellation points and an optimal representation has been derived by using a derandomization algorithm. Since there is one bit that is not used to transmit any information for each constellation point, the transmit power of OFDM systems using constellation extension is much larger than that of OFDM systems with no constellation extension. In [28], PAPR-reduction algorithms 
have been proposed whereby the exterior points of the modulation constellation are modified using linear programming to reduce the peak power of the transmit signal. Due to the constellation extension, these algorithms require an increase in the transmit power and computation complexity at the transmitter.

In this paper, a new algorithm for PAPR reduction is investigated in a probabilistic framework, which consists of two key ingredients. First, a new constellation extension technique is developed whereby the data for each subcarrier can be represented by a point in the original constellation or by an extended point. Second, a new de-randomization algorithm is proposed by applying the so-called method of conditional probability (MCP) [29][30]. The paper also deals with certain modifications to the algorithm that lead to further performance enhancements. These include the use of a selective rotation technique (SR) described in the paper and the application of the coordinate decent optimization (CDO) and the selective mapping algorithms [31][18]. Design examples are presented which demonstrate that the enhanced algorithm offers significant improvements in PAPR reduction and computational complexity over several existing algorithms at the cost of slight increase in the transmit power.

The paper is organized as follows. In Sec. II, a brief description of OFDM systems is given. In Sec. III, the proposed constellation extension scheme is described and the problem is formulated; then the new PAPR-reduction algorithm for the solution of the problem are described. In Sec. IV, various PAPR-reduction algorithms are compared through simulations. Conclusions are drawn in Sec. V.

\section{OFDM SYSTEMS}

In an OFDM system, the available bandwidth $W$ is allocated to $N$ orthogonal subcarriers whose frequencies are seperated by $W / N$. A high-rate data stream is split into $N$ low-rate streams that are transmitted simultaneously through these subcarriers. Each of the subcarriers is independently modulated using phase-shift keying (PSK) or quadrature amplitude modulation (QAM). The modulated signal for each subcarrier is transformed by using the inverse discrete Fourier transform (IDFT) in order to generate the OFDM signal. A cyclic prefix is inserted at the beginning of each OFDM symbol before it is transmitted through the channel. A typical OFDM transmitter is illustrated in Fig. 1 where $\mathrm{S} / \mathrm{P}, \mathrm{P} / \mathrm{S}$, and DAC represent serial-to-parallel, parallel-to-serial, and digital-to-analog converter, respectively, and the block labeled as "Amp." represents a PA. The information bits $D_{k}$ and the modulated symbol $X_{k}$ are referred to as the data point and subsymbol for the $k$ th subcarrier, respectively. Vectors $\mathbf{X}=\left[\begin{array}{lll}X_{0} & \cdots & X_{N-1}\end{array}\right]^{T}$ and $\mathbf{x}=\left[\begin{array}{lll}x_{0} & \cdots & x_{N-1}\end{array}\right]^{T}$ denote the frequency-domain and the time-domain $O F D M$ symbols, respectively. The $n$th element of OFDM symbol $\mathbf{x}$ is given by using the IDFT of $\mathbf{X}$, i.e.,

$$
x_{n}=\frac{1}{N} \sum_{k=0}^{N-1} X_{k} e^{j 2 \pi k n / N} \quad \text { for } n=0, \ldots, N-1
$$

where $x_{n}$ represents the $n$th element of $\mathbf{x}$. Equation (1) can be expressed in matrix form as

$$
\mathbf{x}=\mathbf{Q X}
$$

where $\mathbf{Q}$ is the IDFT matrix whose elements are $q_{n, k}=$ $(1 / N) e^{j 2 \pi k n / N}$. Since an OFDM signal consists of a number of independently modulated subcarriers, when the subsymbols for each subcarrier are added up coherently, the maximum instantaneous power of the OFDM signal could be much larger than its average power. The disparity between the maximum instantaneous power and the average power in OFDM signals is typically quantified in terms of PAPR. For the system shown in Fig. 1, the PAPR of signal $\mathbf{x}$ is defined as

$$
\operatorname{PAPR}=\frac{\|\mathbf{x}\|_{\infty}^{2}}{\mathcal{E}\left[\|\mathbf{x}\|_{2}^{2}\right] / N}
$$

where $\mathcal{E}[\cdot],\|\mathbf{x}\|_{\infty}$, and $\|\mathbf{x}\|_{2}$ denote the expectation of [.], the infinity-norm, and 2-norm of vector $\mathbf{x}$, respectively.

\section{PAPR REDUCTION VIA CONSTELLATION EXTENSION}

\section{A. Constellation Extension Scheme}

For the purpose of illustration, a 16-QAM modulation is assumed for each subcarrier in which case the constellation assumes the form shown in Fig. 2a. The constellation extension scheme for this type of modulation is illustrated in Fig. $2 b$ where any data point with a value greater than or equal to 4 can be represented by a pair of two possible constellation points. For example, data point $D_{k}=15$ (or 1111 in binary form) can be represented either by $X_{k}^{0}=-3-3 j$ or by $X_{k}^{1}=-3+5 j$ where the superscripts of $X_{k}^{0}$ and $X_{k}^{1}$ are used to identify which constellation point is selected to represent $D_{k}$, i.e., $X_{k}^{0}$ indicates that an exterior point of the conventional constellation is used to represent $D_{k}$; on the other hand, $X_{k}^{1}$ indicates that a corresponding extended point is used to represent $D_{k}$. Based on this constellation extension technique, one seeks to reduce the PAPR of the transmit signals by selecting the optimal representation of data points by either the exterior or the extended points. Note that the minimum Euclidean distance between the extended constellaton point and any conventional constellation point is guaranteed to be no less than the minimum distance among the conventional constellation points. As will be demonstrated later, the increase of average transmit power due to the constellation extension is fairly small and, consequently, the BER performance of the system will not be degraded significantly. Note also that the above constellation extension scheme can be easily applied to other modulation constellations without any difficulty.

\section{B. Problem Formulation}

Our objective is to obtain an optimal representation of the data points such that the PAPR of the OFDM symbol $\boldsymbol{X}$ is minimized. This PAPR-reduction problem can be addressed by minimizing the peak power of the transmit signal $\mathbf{x}$. Denoting the number and index set of subcarriers where constellation 
extension is applied as $K$ and $\mathcal{I}=\left\{i_{1}, i_{2}, \ldots, i_{K}\right\}$, respectively, and letting $\overline{\boldsymbol{X}}=\left[\begin{array}{lll}X_{i_{1}} & \cdots & X_{i_{K}}\end{array}\right]^{T}$, the PAPR-reduction problem can be formulated as

$$
\underset{\bar{X}}{\operatorname{minimize}} \max _{0 \leq n \leq N-1}\left|\sum_{k=0}^{N-1} X_{k} e^{\frac{j 2 \pi k n}{N}}\right|
$$$$
\text { subject to: } \quad X_{k} \in\left\{X_{k}^{0}, X_{k}^{1}\right\} \quad \text { for } k \in I
$$

If we let

$$
Y_{k}=\frac{X_{k}^{0}+X_{k}^{1}}{2} \text { and } Z_{k}=\frac{X_{k}^{0}-X_{k}^{1}}{2} \quad \text { for } k \in I
$$

then we have

$$
X_{k}^{0}=Y_{k}+Z_{k} \text { and } X_{k}^{1}=Y_{k}-Z_{k} \quad \text { for } k \in I
$$

and the problem in (4) can be reorganized as

$$
\begin{array}{rl|l}
\underset{\boldsymbol{s}}{\operatorname{minimize}} & \max _{0 \leq n \leq N-1} \mid \sum_{k=0, k \notin I}^{N-1} X_{k} e^{\frac{j 2 \pi k n}{N}} \\
& +\sum_{k=1}^{K} Y_{i_{k}} e^{\frac{j 2 \pi i_{k} n}{N}}+\sum_{k=1}^{K} s_{k} Z_{i_{k}} e^{\frac{j 2 \pi i_{k} n}{N}} \mid
\end{array}
$$$$
\text { subject to: } s_{k} \in\{1,-1\} \text { for } k=1, \ldots, K
$$

where vector $s=\left[\begin{array}{lll}s_{1} & \cdots & s_{K}\end{array}\right]^{T}$ is referred to as the sign vector. The data sets involved in (6) are complex-valued. If we define

$$
c_{n}=\left\{\begin{aligned}
& \operatorname{Re}\left[\sum_{k=0, k \notin I}^{N-1} X_{k} e^{\frac{j 2 \pi k n}{N}}\right.\left.+\sum_{k=1}^{K} Y_{i_{k}} e^{\frac{j 2 \pi i_{k} n}{N}}\right] \quad 0 \leq n \leq N-1 \\
& \operatorname{Im}\left[\sum_{k=0, k \notin I}^{N-1} X_{k} e^{\frac{j 2 \pi k(n-N)}{N}}\right. \\
&\left.\quad+\sum_{k=1}^{K} Y_{i_{k}} e^{\frac{j 2 \pi i_{k}(n-N)}{N}}\right] N \leq n \leq 2 N-1
\end{aligned}\right.
$$

and

$$
d_{n k}= \begin{cases}\operatorname{Re}\left[Z_{i_{k}} e^{\frac{j 2 \pi i_{k} n}{N}}\right] & 0 \leq n \leq N-1 \\ \operatorname{Im}\left[Z_{i_{k}} e^{\frac{j 2 \pi i_{k}(n-N)}{N}}\right] & N \leq n \leq 2 N-1\end{cases}
$$

where $\operatorname{Re}(\cdot)$ and $\operatorname{Im}(\cdot)$ represent the real and imaginary components of the variable, respectively, then the objective function in (6) can be written as

$$
\left|c_{n}+\sum_{k=1}^{K} s_{k} d_{n k}+j c_{n+N}+j \sum_{k=1}^{K} s_{k} d_{n+N, k}\right|
$$

and the problem in (6) can be relaxed to

$$
\underset{s}{\operatorname{minimize}} \max _{0 \leq n \leq 2 N-1}\left|c_{n}+\sum_{k=1}^{K} s_{k} d_{n k}\right|
$$

subject to: $s_{k} \in\{1,-1\}$ for $k=1, \ldots, K(8 \mathrm{~b})$

where the data sets $\left\{c_{n}\right\}$ and $\left\{d_{n k}\right\}$ are real-valued. The relationship between the solutions of the problems in (6) and (8) can be described as follows. If $\tilde{s}$ is the solution of the problem in (6), $\tilde{n}$ is the index $n$ at which the maximum of $\left|c_{n}+\sum_{k=1}^{K} \tilde{s}_{k} d_{n k}+j c_{n+N}+j \sum_{k=1}^{K} \tilde{s}_{k} d_{n+N, k}\right|$ is achieved, $s^{\star}$ is the solution of the problem in (8), $n^{\star}$ is the index $n$ at which the maximum of $\left|c_{n}+\sum_{k=1}^{K} s_{k}^{\star} d_{n k}\right|$ is achieved, then it can be shown that

$$
\begin{aligned}
\mid c_{n^{\star}} & +\sum_{k=1}^{K} s_{k}^{\star} d_{n^{\star} k} \mid \\
& \leq\left|c_{n}+\sum_{k=1}^{K} \tilde{s}_{k} d_{n k}+j c_{n+N}+j \sum_{k=1}^{K} \tilde{s}_{k} d_{n+N, k}\right| \\
& \leq \sqrt{2}\left|c_{n^{\star}}+\sum_{k=1}^{K} s_{k}^{\star} d_{n^{\star} k}\right|
\end{aligned}
$$

It follows from (9) that the solution of the problem in (8) can be regarded as a good approximation of the solution of the problem in (6). For this reason, a good suboptimal solution of the PAPR-reduction problem at hand can be obtained by solving the problem in (8) instead of that in (6).

\section{A PAPR-Reduction Algorithm}

The minimax optimization problem in (8) is an integer programming problem which can be solved by using the method of conditional probability [29][30] and a de-randomization algorithm based on the method of conditional probability will now be proposed. As will be shown, by applying the Chernoff bound to estimate the conditional probability involved and then replacing the many hyperbolic cosine functions produced by the Chernoff-bound estimate with a tight polynomial upper bound, a low computational complexity can be achieved.

1) Chernoff-Bound Based De-Randomization Algorithm: In this subsection, the main results of the Chernoff-bound based de-randomization algorithm proposed in [32] are sketched. Consider sign vector $s=\left[s_{1} \cdots s_{K}\right]$ where $s_{1}, \ldots, s_{K}$ are treated as random variables that can assume the values of 1 or -1 with equal probability. Let $A_{n}^{\lambda}$ be the event that $\left|c_{n}+\sum_{k=1}^{K} d_{n k} s_{k}\right| \geq \lambda$ and $\operatorname{Pr}\left(A_{n}^{\lambda}\right)$ be the probability that event $A_{n}^{\lambda}$ occurs. Let us assume that $\lambda$ is chosen such that

$$
\sum_{n=0}^{2 N-1} \operatorname{Pr}\left(A_{n}^{\lambda}\right)<1
$$

If the first component of the optimal sign vector is taken to be $s_{1}^{\star}=1,{ }^{1}$ then a suboptimal sign vector $s^{\star}$ can be obtained sequentially as

$$
s_{j}^{\star}=\arg \left[\min _{s_{j} \in\{1,-1\}} \sum_{n=0}^{2 N-1} \operatorname{Pr}\left(A_{n}^{\lambda} \mid s_{1}^{\star}, \ldots, s_{j-1}^{\star}, s_{j}\right)\right]
$$

for $j=2, \ldots, K$. It can be shown that the sign vector $s^{\star}=$ $\left[s_{1}^{\star} \cdots s_{K}^{\star}\right]$ obtained using (11) can be regarded as a suboptimal solution for which the objective function in the problem in (8) is guaranteed to be smaller than $\lambda$.

${ }^{1}$ Strictly speaking, the value of $s_{1}$ must be chosen from the set of $\{-1,1\}$. Different values of $s_{1}$ yield different sign vectors that result in different performance. However, simulations show that the results achieved with different values of $s_{1}$ are very close to one another. Therefore, $s_{1}$ is assumed to be equal to one hereafter. 
Since numerical evaluation of the conditional probability is often difficult, an upper bound $U_{n}\left(\lambda, s_{1}, \ldots, s_{j}\right)$ was introduced in [32] for the conditional probability, known as pessimistic estimator [30], such that

$$
\operatorname{Pr}\left(A_{n}^{\lambda} \mid s_{1}, \ldots, s_{j}\right) \leq U_{n}\left(\lambda, s_{1}, \ldots, s_{j}\right)
$$

for $j=1, \ldots, K$, where the conditions

$$
\begin{aligned}
\min _{s_{j} \in\{1,-1\}} & \sum_{n=0}^{2 N-1} U_{n}\left(\lambda, s_{1}, \ldots, s_{j-1}, s_{j}\right) \\
\leq & \sum_{n=0}^{2 N-1} U_{n}\left(\lambda, s_{1}, \ldots, s_{j-1}\right)
\end{aligned}
$$

and

$$
\sum_{n=0}^{2 N-1} U_{n}(\lambda)<1
$$

are satisfied. In effect, working with a pessimistic estimator $U_{n}\left(\lambda, s_{1}, \ldots, s_{j}\right)$ satisfying the conditions in (12a)-(12c), a suboptimal sign vector $s^{\star}$ can be determined sequentially as

$$
\begin{aligned}
s_{j}^{\star}=- & \operatorname{sign}\left[\sum_{n=0}^{2 N-1} U_{n}\left(\lambda, s_{1}^{\star}, \ldots, s_{j-1}^{\star}, 1\right)\right. \\
& \left.-\sum_{n=0}^{2 N-1} U_{n}\left(\lambda, s_{1}^{\star}, \ldots, s_{j-1}^{\star},-1\right)\right]
\end{aligned}
$$

for $j=2, \ldots, K$.

By applying the Chernoff bound [33]

$$
\operatorname{Pr}(Y \geq \delta) \leq e^{-\gamma \delta} E\left(e^{\gamma Y}\right)
$$

to the conditional probability

$$
\begin{aligned}
\operatorname{Pr}\left(\mid c_{n}\right. & \left.+\sum_{k=1}^{K} s_{k} d_{n k}|\geq \lambda| s_{1}, \ldots, s_{j}\right) \\
= & \operatorname{Pr}\left(\sum_{k=j+1}^{K} s_{k} d_{n k} \geq \lambda-c_{n}-\sum_{k=1}^{j} s_{k} d_{n k}\right) \\
& +\operatorname{Pr}\left(-\sum_{k=j+1}^{K} s_{k} d_{n k} \geq \lambda+c_{n}+\sum_{k=1}^{j} s_{k} d_{n k}\right)
\end{aligned}
$$

we obtain

$$
\begin{array}{r}
\operatorname{Pr}\left(\left|c_{n}+\sum_{k=1}^{K} s_{k} d_{n k}\right| \geq \lambda \mid s_{1}, \ldots, s_{j}\right) \leq 2 e^{-\gamma \lambda} \\
\cosh \left(\gamma c_{n}+\gamma \sum_{k=1}^{j} s_{k} d_{n k}\right) \prod_{k=j+1}^{N} \cosh \left(\gamma d_{n k}\right)
\end{array}
$$

Based on the above analysis, a pessimistic estimator can be derived as

$$
\begin{aligned}
& U_{n}\left(\lambda^{\star}, s_{1}, \ldots, s_{j}\right)=2 e^{-\gamma^{\star} \lambda^{\star}} \\
& \quad \cosh \left(\gamma^{\star} c_{n}+\gamma^{\star} \sum_{k=1}^{j} s_{k} d_{n k}\right) \prod_{k=j+1}^{N} \cosh \left(\gamma^{\star} d_{n k}\right)
\end{aligned}
$$

for $j=1, \ldots, K$, where

$$
\begin{aligned}
& \varepsilon=\max _{\substack{0 \leq n \leq 2 N-1\\
}}\left(c_{n}^{2}+\sum_{k=1}^{K} d_{n k}^{2}\right), \lambda^{\star}=\sqrt{2 \varepsilon \log (4 N)}, \\
& \gamma^{\star}=\lambda^{\star} / \varepsilon
\end{aligned}
$$

2) Polynomial Bound for Hyperbolic Cosine: The most computation-intensive operation in the evaluation of upper bound $U_{p}\left(\lambda^{\star}, s_{1}, \ldots, s_{j}\right)$ in (17a) is the evaluation of the hyperbolic cosine functions involved. In what follows, we derive a polynomial upper bound for the hyperbolic cosine function on a given interval that will eventually lead to a polynomial-bound based pessimistic estimator.

It follows from (16) that

$$
\begin{aligned}
\operatorname{Pr}\left(\gamma\left|c_{n}+\sum_{k=1}^{K} s_{k} d_{n k}\right| \geq \gamma \lambda\right) \leq 2 e^{-\gamma \lambda} \\
\max _{\substack{n=0, \ldots, 2 N-1\\
}}\left[\cosh \left(\gamma c_{n}\right) \prod_{k=1}^{K} \cosh \left(\gamma d_{n k}\right)\right]
\end{aligned}
$$

For example, for $N=64$ we have $\gamma=20$ and $\gamma \lambda=11$. In such a case, (18) becomes

$$
\operatorname{Pr}\left(\gamma\left|c_{n}+\sum_{k=1}^{K} s_{k} d_{n k}\right| \geq \gamma \lambda\right) \leq P_{n}
$$

where

$$
\begin{aligned}
P_{n} & =2 e^{-11} \max _{n=0, \ldots, 2 N-1}\left[\cosh \left(\gamma c_{n}\right) \prod_{k=1}^{K} \cosh \left(\gamma d_{n k}\right)\right] \\
& =0.0025
\end{aligned}
$$

We stress that the bound derived in (19) is not tight and computer simulations have indicated that the actual interval into which the value of $\gamma\left|c_{n}+\sum_{k=1}^{K} s_{k} d_{n k}\right|$ falls is considerably smaller than $[0,11]$. Based on these observations, we seek a polynomial upper bound $g(x)=1+k_{1} x^{2}+k_{2} x^{4}$ for $\cosh (x)$ with $0 \leq x \leq M$, i.e.,

$$
\cosh (x) \leq 1+k_{1} x^{2}+k_{2} x^{4} \quad \text { for } 0 \leq x \leq M
$$

If we define

$$
\begin{gathered}
G_{n}\left(\lambda, s_{1}, \ldots, s_{j}\right)=2 e^{-\gamma \lambda} g\left(\gamma c_{n}+\gamma \sum_{k=1}^{j} s_{k} d_{n k}\right) \\
\prod_{k=j+1}^{K} g\left(\gamma d_{n k}\right)
\end{gathered}
$$

then (17), (20), and (21) imply that

$$
\operatorname{Pr}\left(A_{n}^{\lambda} \mid s_{1}, \ldots, s_{j}\right) \leq G_{n}\left(\lambda, s_{1}, \ldots, s_{j}\right)
$$

which is the condition in (12a) for $G_{n}\left(\lambda, s_{1}, \ldots, s_{j}\right)$. In addition, if the polynomial $g(x)$ satisfies the inequality

$$
g(a) g(b) \geq \frac{g(a+b)+g(a-b)}{2}
$$


for any $a$ and $b$, then we have

$$
\begin{aligned}
& \sum_{n=0}^{2 N-1} G_{n}\left(\lambda, s_{1}, \ldots, s_{j-1}\right) \\
& \quad \geq \min _{s_{j} \in\{1,-1\}} \sum_{n=0}^{2 N-1} G_{n}\left(\lambda, s_{1}, \ldots, s_{j-1}, s_{j}\right)
\end{aligned}
$$

which is the condition in (12b) for $G_{n}\left(\lambda, s_{1}, \ldots, s_{j}\right)$. It can be verified that polynomial bound $g(x)$ satisfies (23) if its coefficients are chosen such that

$$
k_{1}^{2}-6 k_{2} \geq 0
$$

To identify the optimal values of parameters $k_{1}$ and $k_{2}$, we introduce an upper bound for the difference $g(x)-\cosh (x)$ as

$$
g(x)-\cosh (x) \leq \delta \quad \text { for } 0 \leq x \leq M
$$

and $k_{1}$ and $k_{2}$ are determined by minimizing $\delta$ subject to the constraints in (20), (25), (26), and $k_{1} \geq 0, k_{2} \geq 0$. The nonlinear constraint in (25) in conjunction with the nonnegativity constraints $k_{1} \geq 0$ and $k_{2} \geq 0$ defines the shaded region in Fig. 3. This nonconvex region can be approximated by the triangular region defined by a tangent line of the parabola $k_{2}=k_{1}^{2} / 6$ and the $k_{1}$-axis where the triangular region is convex and can be described by the constraint

$$
\frac{c k_{1}}{3}-k_{2}-\frac{c^{2}}{6} \geq 0
$$

Replacing the nonlinear constraint in (26) by the linear constraint in (27), the optimal parameters $k_{1}$ and $k_{2}$ for polynomial $g(x)$ can be determined by solving the linear programming (LP) problem

\section{minimize $\delta$}

subject to:

$$
\begin{aligned}
& k_{1} \geq 0, k_{2} \geq 0, \\
& \frac{c k_{1}}{3}-k_{2}-\frac{c^{2}}{6} \geq 0 \\
& k_{1} x^{2}+k_{2} x^{4}-\cosh (x)+1 \geq 0 \\
& \quad \text { for } 0 \leq x \leq M \\
& -k_{1} x^{2}-k_{2} x^{4}+\cosh (x)-1-\delta \geq 0 \\
& \text { for } 0 \leq x \leq M
\end{aligned}
$$

where the parameter vector of the LP problem is $\left[\begin{array}{lll}\delta & k_{1} & k_{2}\end{array}\right]^{T}$ and variable $x$ assumes values in interval $[0, M]$. For example, with $M=6$ and $c=0.88$, solving the LP problem in (28) gives $k_{1}=0.8844$ and $k_{2}=0.1303$.

For $M=6$, it can be shown that if the values of $\gamma$ and $\lambda$ in (21) are chosen such that

$$
\begin{aligned}
& \varepsilon=\max _{0 \leq n \leq 2 N-1} \sum_{k=1}^{K}\left(c_{n}^{2}+d_{n k}^{2}\right), \lambda^{\star}=\sqrt{3.538 \varepsilon \log (4 N)}, \\
& \gamma^{\star}=0.565 \lambda^{\star} / \varepsilon
\end{aligned}
$$

then

$$
\begin{gathered}
G_{n}\left(\lambda^{\star}, s_{1}, \ldots, s_{j}\right)=2 e^{-\gamma^{\star} \lambda^{\star}} g\left(\gamma^{\star} c_{n}+\gamma^{\star} \sum_{k=1}^{j} s_{k} d_{n k}\right) \\
\prod_{k=j+1}^{K} g\left(\gamma^{\star} d_{n k}\right)
\end{gathered}
$$

satisfies the condition in (12c) and, therefore, $G_{n}\left(\lambda^{\star}, s_{1}, \ldots, s_{j}\right)$ can be used as a pessimistic estimator.

The polynomial-bound based pessimistic estimator in (30) can be used in conjunction with (13) to obtain a suboptimal solution $s^{\star}$ for problem (8). Using (30), Eq. (13) becomes

$$
\begin{aligned}
s_{j}^{\star}=- & \operatorname{sign}\left[\sum_{n=0}^{2 N-1} G_{n}\left(\lambda^{\star}, s_{1}^{\star}, \ldots, s_{j-1}^{\star}, 1\right)\right. \\
& \left.-\sum_{n=0}^{2 N-1} G_{n}\left(\lambda^{\star}, s_{1}^{\star}, \ldots, s_{j-1}^{\star},-1\right)\right]
\end{aligned}
$$

for $j=2, \ldots, K$. Using (5b) and (31), the optimized OFDM symbol $\boldsymbol{X}^{\star}$ can be obtained as

$$
X_{k}^{\star}= \begin{cases}X_{k} & \text { for } k \notin I \\ Y_{k}+s_{l}^{\star} Z_{k} & \text { for } k \in I\end{cases}
$$

where $l$ is the index of element $k$ in set $I$ in the case that $k \in I$. From (4), (8), and (9), we can write

$$
\max _{0 \leq n \leq N-1}\left|\sum_{k=0}^{N-1} X_{k}^{\star} e^{\frac{j 2 \pi k n}{N}}\right|^{2}<2 \lambda^{\star 2}
$$

In other words, the peak power of optimized OFDM symbol $\boldsymbol{X}^{\star}$ is guaranteed to be smaller than $2 \lambda^{\star 2}$. An algorithm based on the above solution method is summarized in Table 1.

3) Computational Complexity: The computation required by (31) in the proposed algorithm entails $12 \mathrm{NK}$ real multiplications, $6 N K$ real additions, and $K$ sign function evaluations. Note that most of the multiplications involved in these algorithms entail multiplication by a constant, which can be carried out efficiently [34]-[35]. Consequently, the actual computational efficiency of the proposed algorithms is much better than the above numbers would suggest, which makes a fair comparison with other algorithms a somewhat complicated matter. To avoid this difficulty, the computational complexity of the various PAPR algorithms was measured in terms of the CPU time required by the simulations described in Sec. IV.

\section{Enhancement for the Proposed Algorithms}

The performance of the proposed algorithm can be improved further through several enhancement techniques as described below.

1) Selective Rotations: The objective function in (6a) remains unchanged if all complex-valued terms are rotated by an angle $\theta$ since

$$
\begin{aligned}
& \left|\sum_{k=0, k \notin I}^{N-1} X_{k} e^{\frac{j 2 \pi k n}{N}}+\sum_{k=1}^{K}\left(Y_{i_{k}}+s_{k} Z_{i_{k}}\right) e^{\frac{j 2 \pi i_{k} n}{N}}\right| \\
& =\left|e^{j \theta}\left[\sum_{k=0, k \notin I}^{N-1} X_{k} e^{\frac{j 2 \pi k n}{N}}+\sum_{k=1}^{K}\left(Y_{i_{k}}+s_{k} Z_{i_{k}}\right) e^{\frac{j 2 \pi i_{k} n}{N}}\right]\right|
\end{aligned}
$$

On the other hand, this rotation leads to a different set of $d_{n k}$ which can be obtained using (7) with $X_{k}, Y_{i_{k}}$, and $Z_{i_{k}}$ replaced by $e^{j \theta} X_{k}, e^{j \theta} Y_{i_{k}}$, and $e^{j \theta} Z_{i_{k}}$, respectively. If we use the parameters $d_{n k}$ generated by $R$ different rotation angles $\theta_{0}, \theta_{1}, \ldots, \theta_{R-1}$ with $\theta_{0}=0$, then we can obtain 
$R$ suboptimal sign vectors $s_{0}^{\star}, s_{1}^{\star}, \ldots, s_{R-1}^{\star}$ from which the best sign vector can be identified by comparing the performance of the corresponding suboptimal solutions. Since the set of rotation angles includes $\theta=0$, the suboptimal solution obtained using the SR algorithm is always superior to the suboptimal solution of the de-randomization algorithm described in Table 1. Note that for any given sign vector, the transmit signal for each subcarrier satisfies the constellation extension scheme in Fig. $2 \mathrm{~b}$ and, therefore, no side information needs to be transmitted along with the useful information.

2) Coordinate Descent Optimization: If we define

$$
f(\mathbf{s})=\max _{0 \leq n \leq 2 N-1} f_{n}(\mathbf{s})
$$

where

$$
f_{n}(\mathbf{s})=\left|c_{n}+\sum_{k=1}^{K} s_{k} d_{n k}\right| \quad \text { for } n=0, \ldots, 2 N-1
$$

with $\mathbf{s}=\left[s_{1} \cdots s_{K}\right]$ and $s_{k} \in[1,-1]$ for $k=1, \ldots, K$, then the idea of coordinate descent optimization [36] can be applied to reduce the value of $f(\mathbf{s})$ iteratively as follows. Suppose only one element of the sign vector $\mathbf{s}$ is allowed to switch at each iteration. First, the value of $f_{n}(\mathbf{s})$ after the sign switch of element $s_{k_{c}}$ can be obtained as

$$
f_{n}\left(\mathbf{s}, k_{c}\right)=\left|c_{n}+\sum_{k=1, k \neq k_{c}}^{K} s_{k} d_{n k}-s_{k_{c}} d_{n k_{c}}\right|
$$

for $n=0, \ldots, 2 N-1$ and $k_{c}=1, \ldots, K$. Next, the change in the value of $f$ can be obtained as

$$
\Delta_{f}\left(k_{c}\right)=f(\mathbf{s})-\max _{0 \leq n \leq 2 N-1} f_{n}\left(\mathbf{s}, k_{c}\right)
$$

for $k_{c}=1, \ldots, K$. If we define

$$
\hat{k}_{c}=\arg \left[\max _{1 \leq k_{c} \leq K} \Delta_{f}\left(k_{c}\right)\right]
$$

and $\Delta_{f}\left(\hat{k}_{c}\right) \leq \epsilon$, where $\epsilon$ is a predefined tolerance, then a local minimum of function $f(\mathbf{s})$ is achieved and the algorithm terminates. Otherwise, the steepest descent direction for function $f(\mathbf{s})$ can be found as $-s_{\hat{k}_{c}}$. Therefore, the sign vector can be

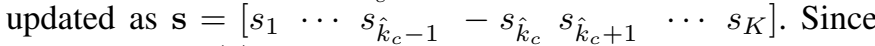
the value of $f(\mathbf{s})$ is constantly reduced at each iteration, the CDO technique can be applied to enhance the performance of the de-randomization algorithm proposed in Sec. III. C.3. A step-by-step description of the CDO algorithm is given in Table 2.

Note that the $\mathrm{CDO}$ algorithm can be generalized by allowing multiple elements of the sign vector $\mathbf{s}$ to change in each iteration. This technique tends to improve the performance at the cost of an increased computational complexity.

3) Improved Performance Using the SM Algorithm: In the proposed algorithm, only one data set has been utilized for PAPR reduction. The performance can be improved by combining the proposed algorithm with the SM algorithm as illustrated in Fig. 4. First, multiple candidate data sets are generated at the transmitter. Second, for each of the data sets the proposed de-randomization algorithm is applied and the one with the least PAPR is selected. Third, the proposed SR and CDO algorithms are applied to the data set selected in the second stage for further PAPR reduction.

\section{Simulations}

The proposed algorithm was applied to a system with 64 subcarriers and the PAPR-reduction performance was evaluated and compared with that of the SM and SCE algorithms proposed in [18] and [27], respectively. The signal was oversampled by a factor of 2 using zero padding in the frequency domain before the application of the PAPRreduction algorithms. In order to approximate the analog signal accurately [37]-[32], the sampling rate was further increased by a factor of 4 by using a root-raised cosine (RRC) filter with a rolloff factor of 0.12 . The performance was evaluated by measuring the output signal of the RRC filter. For the SM algorithm, the number of candidate sequences is denoted as $U$. For the proposed SR algorithm, the number of rotations is denoted as $R$. The rotation angle $\theta$ was set to the values $0, \pi / R, \ldots,(R-2) \pi / R,(R-1) \pi / R$. For the proposed CDO algorithm, the number of elements of the sign vector that can be changed at each optimization iteration is denoted as Bit. Example 1: The constellation extension scheme adopted for the proposed algorithm is shown in Fig. 2b where only exterior points are used for PAPR reduction. Satistically, the number of subcarriers where constellation extenstion is applicable is three quarters the number of subcarriers, namely, $K \doteq \frac{3}{4} N$. The clipping probabilities versus various power threshold values are plotted as the solid curves in Fig. 5. For the SM algorithm, a 16-QAM constellation was adopted. For the sake of comparison, the clipping probabilities obtained using the SM algorithm and for the OFDM signal with no PAPR reduction are plotted in the same figure as dot-dashed curves. First, it can be observed from Fig. 5 that by combining the proposed algorithm with the SR algorithm, a significant PAPR reduction can be achieved. For example, for a clipping probability of $10^{-3}$, a 1.4- and 2.8-dB improvement can be obtained by using the proposed algorithm with $R=1$ and $R=4$, respectively, over the OFDM signal with no PAPR reduction. Note that the power increase associated with the proposed algorithm is approximately $0.41 \mathrm{~dB}$ above the average power of the OFDM signal with no PAPR reduction. Second, it can be observed from Fig. 6 that by combining the proposed algorithm with the CDO algorithm, significant performance improvement can be achieved relative to that achieved with the SM algorithm. For example, by using the proposed algorithm with CDO and assuming a clipping probability of $10^{-3}$, an improvement of $0.9 \mathrm{~dB}$ can be achieved relative to the performance achieved with the SM algorithm with $U=16$. Note that, in such a case, the power increase with respect to the average power is approximately $0.64 \mathrm{~dB}$. Third, it can be observed from Fig. 7 that the performance can be further improved by combining the proposed algorithm with the SM algorithm. For example, by using the combined algorithm with $U=4, R=2$, Bit $=1$, a 1.4-dB improvement can be achieved over the SM algorithm with $U=16$.

Example 2: The performance of the algorithms using various constellation extension schemes was compared and the results 
are plotted in Fig. 8. The clipping probabilities for systems using the schemes in Fig. 2b and Fig. 9 [27] versus various power threshold values are plotted in Fig. 8 as dashed and solid curves, respectively. It can be observed that significant improvement can be achieved by using the proposed algorithm with the scheme in Fig. $2 b$ over the SCE algorithms with the scheme in Fig. 9.

The proposed and the SCE algorithms apply constellation extension as illustrated in Figs. 2(b) and 9, respectively, to reduce the PAPR. As can be seen in Figs. 9 and 2(a), the size of the constellation in Fig. 9 is twice that of the 16QAM constellation in Fig. 2(a). For both of the constellation extension schemes shown in Figs. 2(b) and 9, one constellation point represents 4 information bits. Therefore, an OFDM system using the proposed algorithm with the constellation in Fig. 2(b) has the same effective transmission rate as a system using the SCE algorithm with the constellation in Fig. 9, that is, 4 bits per subcarrier.

The BER performance of OFDM systems using the constellation schemes in Figs. (2b) and 9 was investigated assuming an AWGN channel. In order to achieve comparable BER performance, the minimum distances between the nearest constellation points for the constellations in Figs. 2(b) and 9 should be equal. In such a case, the average transmit power achieved with the SCE algorithm and the constellation in Fig. 9 is $3 \mathrm{~dB}$ higher than that achieved with the 16-QAM constellation. This increase is much higher than that achieved with the scheme in Fig. 2b, which is about $0.64 \mathrm{~dB}$.

The computational complexities of the proposed algorithm is compared with those of existing algorithms in Table 3 where the performance of each algorithm is quantified in terms of its PAPR-reduction improvement in $\mathrm{dB}$ over the OFDM signal with no PAPR reduction for a clipping probability of $10^{-3}$. The computational complexity of the algorithms is measured in terms of the ratio of the CPU time required for each algorithm to that of the SM algorithm with $U=16$, for which the CPU time was normalized to unity. It can be observed that by combining the proposed algorithm with the SR, CDO, and SM algorithms, improved performance can be achieved with less computation with respect to the performance and computation required by some existing PAPR-reduction algorithms.

From Table 3, the following conclusions can be drawn:

- By combining the proposed algorithm with the SR, CDO, and SM algorithms, improved performance and reduced computation can be achieved with respect to those of the SM algorithm in [18].

- The proposed algorithm offers a tradeoff between performance and computational complexity. For example, if the computational resources available are very limited, then the performance offered by the proposed algorithm is marginal; if additional computation resources are available, then improved performance can be achieved by using a combination of the proposed algorithm, SR algorithm, or the CDO algorithm; if additional side information is available, then the performance can be further improved by also using the SM algorithm.

\section{CONCLUSions}

A new PAPR-reduction algorithm for OFDM systems via constellation extension has been proposed based on a conditional probability method whereby the evaluation of the hyperbolic cosine functions involved in the Chernoff bound of the conditional probability is replaced by a tight polynomial bound that leads to reduced computational complexity. The performance of the algorithm can be significantly improved by incorporating the selective rotation technique or by combining the algorithm with the coordinate descent optimization and selective mapping algorithms. Simulations have demonstrated that the enhanced algorithm outperforms the selective mapping algorithm in [18] and the symmetric constellation extension algorithm in [27] in terms of PAPR reduction and computational complexity.

\section{REFERENCES}

[1] J. Bingham, "Multicarrier modulation for data transmission: an idea whose time has come," IEEE Commun. Mag., pp. 5-14, May 1990.

[2] R. V. Nee and R. Prasad, OFDM for Wireless Multimedia Communications, Artech House Publisher, 2000.

[3] W. Y. Zou and Y. Wu, "COFDM: an overview," IEEE Trans. Broadcast., vol. 41, pp. 1-8, Mar. 1995.

[4] ETSI, "Radio broadcasting systems: digital audio broadcasting to mobile, portable and fixed receivers," European Telecommunication Standard, ETS 300-401, Feb. 1995

[5] ETSI, "Digital video broadcasting: framing structure, channel coding, and modulation for digital terrestrial television," European Telecommunication Standard, ETS 300-744, Aug. 1997.

[6] IEEE 802.11, "IEEE standard for wireless LAN medium access control (MAC) and physical layer (PHY) specifications," Nov. 1997.

[7] C. Rapp, "Effects of HPA-nonlinearity on a 4-DPSK/OFDM signal for a digital sound boradcasing system," Proc. Second Euro. Satellite Commun., pp. 179-184, Oct. 1991.

[8] S. Merchan, A. G. Armada, and J. L. Garcia, "OFDM performance in amplifier nonlinearity," IEEE Trans. Broadcast., vol. 44, pp. 106-114, Mar. 1998.

[9] D. J. G. Mestdagh, P. Spruyt, and B. Biran, "Analysis of clipping effect in DMT-based ADSL systems," Proc. IEEE Int. Conf. Commun., pp. 293-300, 1994.

[10] X. Li and L. J. Cimini, Jr., "Effects of clipping and filtering on the performance of OFDM," IEEE Commun. Lett., vol. 2, pp. 131-133, May 1998.

[11] H. Ochiai and H. Imai, "Performance analysis of deliberately clipped OFDM signals," IEEE Trans. Commun., vol. 50, pp. 89-101, Jan. 2002.

[12] A. E. Jones and T. A. Wilkinson, "Combined coding for error control and increased robustness to system nonlinearities in OFDM," Proc. IEEE Conf. Vehicular Tech., pp. 904-908, May 1996.

[13] R. D. J. Van Nee, "OFDM codes for peak-to-average power reduction and error correction," Proc. IEEE Conf. Global Telecomm., pp. 740-744, Nov. 1996.

[14] J. A. Davis and J. Jedwab, "Peak-to-mean power control and error correction for OFDM transmission using Golay sequences and ReedMuller codes," Electron. Lett., vol. 33, pp. 267-268, Nov. 1997.

[15] K. G. Paterson and V. K. Tarokh, "On the existence and construction of good codes with low peak-to-average power ratios," IEEE Trans. Inform. Theory, vol. 46, pp. 1974-1987, Sep. 2000.

[16] C. Robing and V. K. Tarokh, "A construction of OFDM 16-QAM sequences having low peak powers," IEEE Trans. Inform. Theory, vol. 47, pp. 2091-2094, Jul. 2001.

[17] V. .C. Chan, R. Venkataramani, and V. K. Tarokh, "A new construction of 16-QAM Golay complementary sequences," IEEE Trans. Inform. Theory, vol. 49, pp. 2953-2959, Nov. 2003.

[18] M. Breiling, S. Mueller-Weinfurtner, and J. Huber, "Distortionless reduction of peak power without explicit side information," Proc. IEEE Conf. Global Telecomm., pp. 1494-1498, 2000.

[19] S. Mueller, R. Baeuml, R. Fischer, and J. Huber, "OFDM with reduced peak-to-average power ratio by multiple signal representation," Annals of Telecommunications, Feb. 1997. 
[20] H. Ochiai and H. Imai, "Performance of the deliberate clipping with adaptive symbol selection for strictly band-limited OFDM systems," IEEE J. Select. Areas Commun., vol. 18, pp. 2270-2277, Nov. 2000.

[21] H. Ochiai, "A novel trellis-shaping design with both peak and average power reduction for OFDM systems," IEEE Trans. Commun., vol. 52, pp. 1916-1926, Nov. 2004.

[22] M. Chen and O. M. Collins, "Trellis pruning for peak-to-average power ratio reduction," Proc. International Symposium on Information Theory, pp. 1261-1265, 2005.

[23] J. Tellado and J. M. Cioffi, "PAR reduction in multicarrier transmission systems," ANSI document, TIE1.4 Technical Subcommittee, contribution number 97-367, Dec. 1997.

[24] J. Tellado, Peak to Average Power Reduction for Multicarrier Modulation, Ph. D. Dissertation Stanford, CA: Stanford Univ., 2000.

[25] D. Jones, "Peak power reduction in OFDM and DMT via active channel modification," Proc. 33rd Asilomar Conf. Signals, Systems and Computers, pp. 1076-1079, 1999.

[26] B. S. Krongold and D. L. Jones, "PAR reduction in OFDM via active constellation extension," Proc. IEEE Int. Conf. Acoustics, Speech, and Signal Processing, vol. 4, pp. IV525-IV528, Apr. 2003.

[27] M. Sharif and B. Hassibi, "A deterministic algorithm that achieves the PMEPR of clogn for multicarrier signals," Proc. IEEE Int. Conf. Acoustics, Speech, and Signal Processing, vol. 4, pp. 540-543, Oct. 2003.

[28] Y. J. Kou, W.-S. Lu, and A. Antoniou "New peak-to-average power-ratio reduction algorithms for multicarrier communications," IEEE Trans. on Circuits and Systems I: Regular Papers, vol. 51, pp. 1790-1800, Sep. 2004

[29] J. Spencer, Ten Lectures on the Probabilistic Method, SIAM, Philadelphia, 1987.

[30] P. Raghavan, "Probabilistic construction of deterministic algorithms approximating packing integer program," J. Computer and System Sciences, vol. 37, pp. 130-143, 1988.

[31] P. M. Pardalos and S. Jha "Complexity of uniqueness and local search in quadratic 0-1 programming," Operations Research Letters, vol. 11 , pp. 119-123, 1992.

[32] M. Sharif and B. Hassibi, "Existence of codes with constant PMEPR and related design," IEEE Trans. Signal Processing, vol. 52, pp. 2836-2846, Oct. 2004.

[33] J. G. Proakis, Digital Communications, 4th ed., McGraw-Hill, 2000.

[34] K. Wiatr and E. Jamro, "Constant coefficient multiplication in FPGA structures," Proc. Euromicro Conference, vol. 1, pp. 252-259, Sep. 2000

[35] Y. Wang and K. Roy, "CSDC: a new complexity reduction technique for multiplierless implementation of digital FIR filters," IEEE Trans. on Circuits and Systems I: Regular Papers, vol. 52, pp.1845-1853, Sep. 2005.

[36] J. Luo, K. Pattipati, and P. Willett, "A class of coordinate descent methods for multiuser detection," Proc. IEEE Int. Conf. Acoustics, Speech, and Signal Processing, vol. 5, pp. 2853-2856, Jun. 2000.

[37] C. Tellambura, "Phase optimization criterion for reducing peak-toaverage power ratio for OFDM," Electron. Lett., pp. 169-170, Jan. 1998.

[38] H. Wunder and H. Boche, "Peak value estimation of bandlimited signals from their samples, noise enhancement, and a local characterization in the neighborhood of an extremum," IEEE Trans. Signal Processing, vol. 51, pp. 771-780, Mar. 2003.

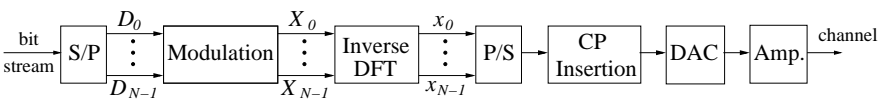

Fig. 1. An OFDM transmitter.

\section{TABLE I}

The Proposed Algorithm

\begin{tabular}{l}
\hline Step 1 \\
Input the OFDM symbol $\boldsymbol{X}=\left[\begin{array}{lll}X_{1} & \cdots & X_{N}\end{array}\right]$. \\
Step 2 \\
For $k \in I$, compute $Y_{k}$ and $Z_{k}$ using (5a). \\
Compute $c_{n}$ and $d_{n k}$ using (7). \\
Evaluate parameters $\varepsilon, \lambda^{\star}$, and $\gamma^{\star}$ using (30). \\
Step 3 \\
Determine the sign vector $\boldsymbol{s}^{\star}=\left[\begin{array}{lll}s_{1}^{\star} & \cdots & s_{K}^{\star}\end{array}\right]$ with $s_{1}^{\star}=1$ using (31). \\
The optimized OFDM symbol $\boldsymbol{X}^{\star}$ can be obtained using (32). \\
\hline
\end{tabular}

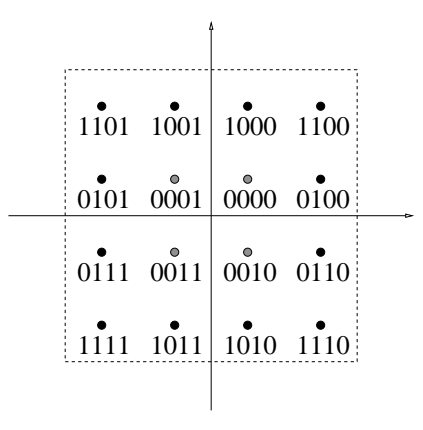

(a)

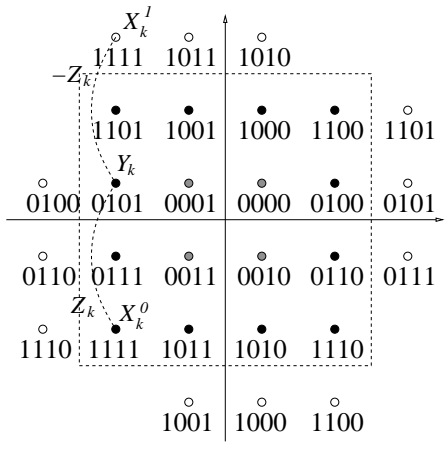

(b)

Fig. 2. (a) 16-QAM constellation with Gray code bit mapping. (b) extension of 16-QAM constellation.

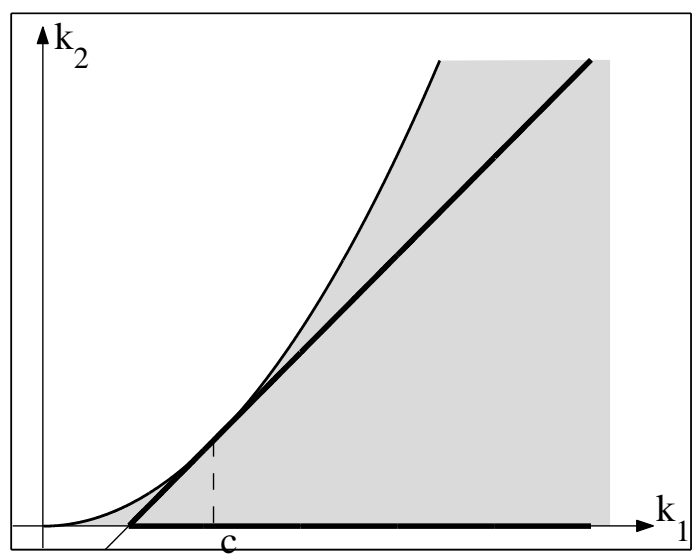

Fig. 3. An approximation for the nonconvex constraint.

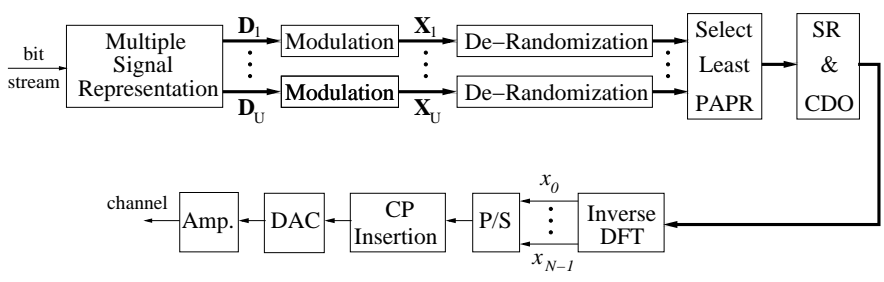

Fig. 4. Combination of the proposed and the SM algorithms.

\section{TABLE II}

CoOrdinate Descent Optimization Algorithm

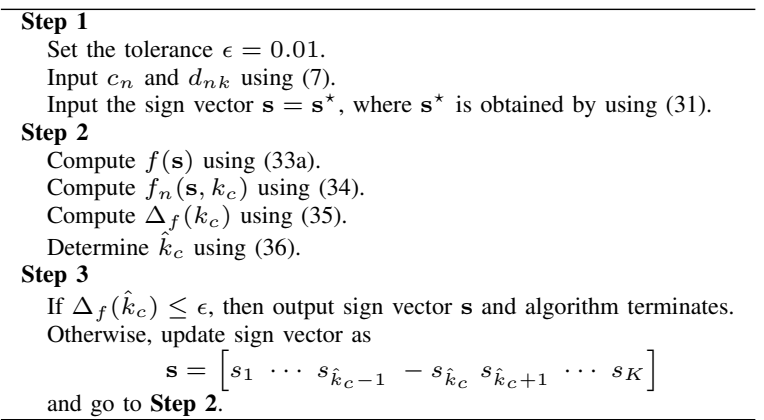


TABLE III

Performance And Complexity of PAPR-Reduction Algorithms

\begin{tabular}{|c|c|c|c|c|c|c|c|}
\hline \multirow[t]{3}{*}{ Algorithms } & \multirow{3}{*}{$\begin{array}{c}\text { SM } \\
\text { Algorithm } \\
U=16\end{array}$} & \multicolumn{6}{|c|}{$\begin{array}{c}\text { Proposed PAPR-Reduction Algorithm } \\
\end{array}$} \\
\hline & & \multicolumn{2}{|c|}{$\begin{array}{c}\text { No SM }(U=1) \\
\text { No } C D O(B i t=0)\end{array}$} & \multicolumn{2}{|c|}{$\begin{array}{l}\text { No SM }(U=1) \\
\text { CDO }(B i t=1)\end{array}$} & \multicolumn{2}{|c|}{$\begin{array}{c}\operatorname{SM}(U=4) \\
\operatorname{CDO}(B i t=1)\end{array}$} \\
\hline & & $\begin{array}{c}\text { SR } \\
(R=1)\end{array}$ & $\begin{array}{c}\text { SR } \\
(R=4)\end{array}$ & $\begin{array}{c}\text { SR } \\
(R=1)\end{array}$ & $\begin{array}{c}\text { SR } \\
(R=4)\end{array}$ & $\begin{array}{c}\text { SR } \\
(R=1)\end{array}$ & $\begin{array}{c}\text { SR } \\
(R=2)\end{array}$ \\
\hline $\begin{array}{l}\text { Performance } \\
\text { Gain }(\mathrm{dB})\end{array}$ & 3.4 & 1.45 & 2.85 & 3.8 & 4.4 & 4.8 & 5 \\
\hline $\begin{array}{l}\text { Normalized } \\
\text { CPU Time }\end{array}$ & 1 & 0.10 & 0.45 & 0.41 & 1.65 & 0.83 & 1.25 \\
\hline
\end{tabular}

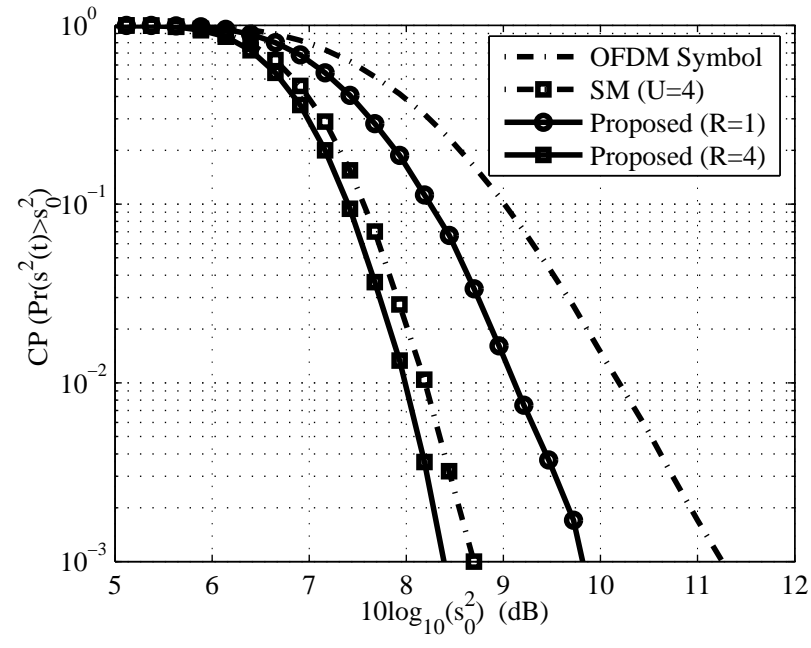

Fig. 5. Performance comparison of the SM and the de-randomization algorithm with SR.

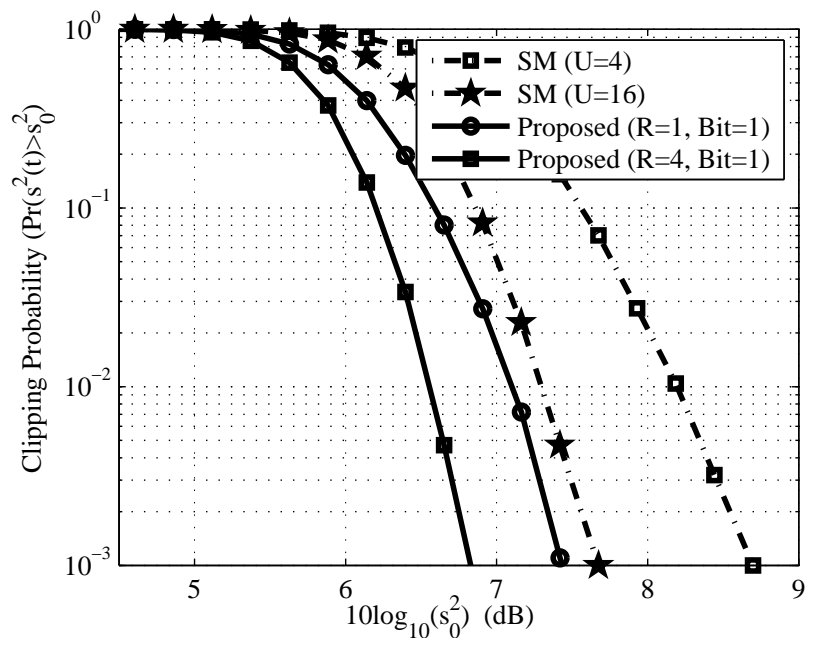

Fig. 6. Performance comparison of the SM and the de-randomization algorithm with SR and CDO.

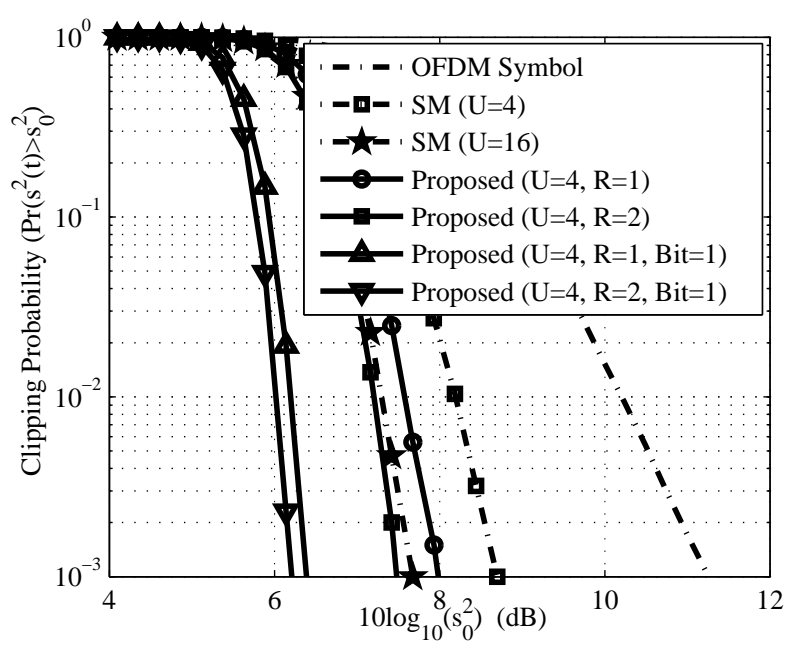

Fig. 7. Performance comparison of the $\mathrm{SM}$ and the de-randomization algorithm which combines with SR, CDO and SM.

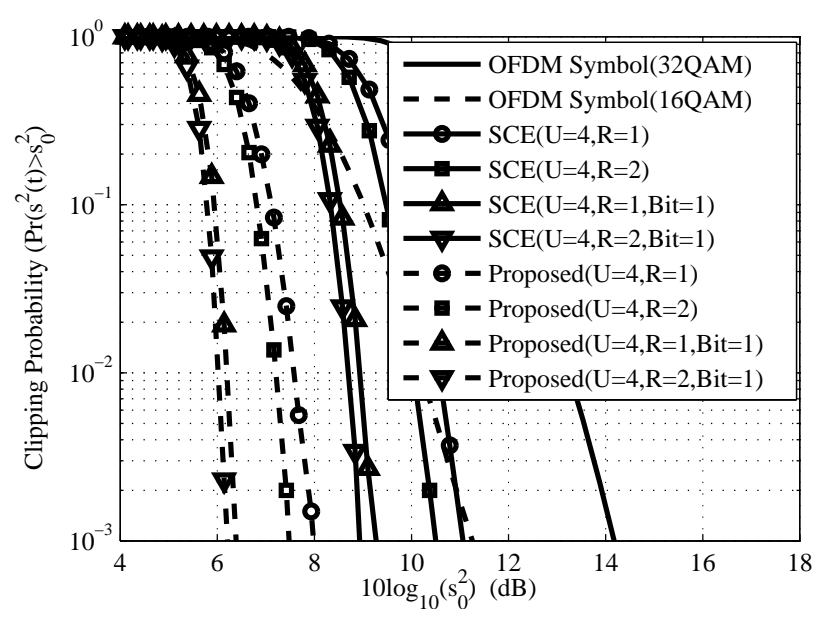

Fig. 8. Performance comparison of the de-randomization algorithm with SR, $\mathrm{CDO}$, and SM using various constellation extension schemes. 


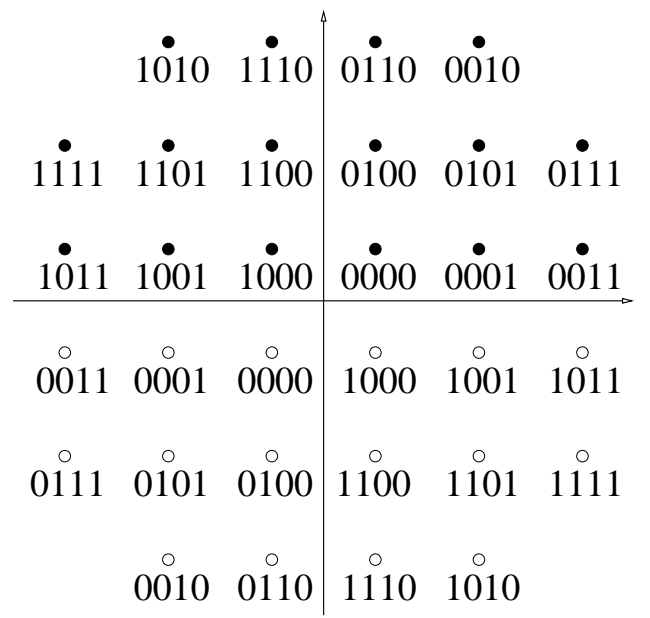

Fig. 9. 32-QAM constellation using symmetric constellation extension scheme. 